\title{
EFEITO DA EXPLORAÇÃO DE MADEIRA E DOS TRATAMENTOS SILVICULTURAIS NO AGRUPAMENTO ECOLÓGICO DE ESPÉCIES
}

\author{
Celso Paulo de Azevedo*, Carlos Roberto Sanquetta**, José Natalino Macedo Silva***, \\ Sebastião do Amaral Machado**** \\ *Eng. Florestal, Dr., Embrapa Amazônia Ocidental - celso@cpaa.embrapa.br \\ **Eng. Florestal, Ph.D., Depto. de Ciências Florestais, UFPR - sanqueta@ufpr.br \\ ***Eng. Florestal, Ph.D., Embrapa Amazônia Oriental - natalino.silva@cgiar.org \\ ****Eng. Florestal, Ph.D., Depto. de Ciências Florestais, UFPR, Pesquisador 1A do CNPq - samachado@ufpr.br
}

Recebido para publicação: 26/04/2007 - Aceito para publicação: 09/09/2007

\begin{abstract}
Resumo
Este estudo foi conduzido com dados do Projeto Bom Manejo (Embrapa-CPATU/CIFOR/ITTO), na Jari, Vitória do Jari, Amapá. O delineamento é em blocos ao acaso, sendo testadas três intensidades de exploração $(15 \%, 25 \%$ e $35 \%)$ do volume das árvores com dap $>60 \mathrm{~cm}$, seguidas de quatro níveis de redução de área basal original $(0 \%, 30 \%, 50 \%$ e $70 \%)$. São 40 parcelas permanentes (36 exploradas e 4 não-exploradas). O povoamento foi medido em 1984, explorado em 1985 e remedido em 1986, 1988, 1990, 1994, 1996 e 2004. Foram medidas todas árvores com dap $\geq 20 \mathrm{~cm}$. O processo envolveu três estágios: (1) análise de cluster para fazer o agrupamento das espécies mais populosas; (2) análise discriminante, para adicionar as menos populosas aos grupos existentes; (3) estágio subjetivo, em que as espécies com poucos dados foram alocadas nos grupos existentes. As variáveis características de cada espécie na análise de cluster foram: Incremento Periódico Anual em diâmetro (IPA $\left.A_{D A P}\right)$ médio e percentil $95 \%$ da distribuição de freqüência cumulativa dos DAPs. Concluiu-se que a exploração de árvores comerciais e a aplicação dos tratamentos silviculturais estimularam o crescimento das plantas já estabelecidas e, diferentemente do esperado, não favoreceram o crescimento de espécies pioneiras.

Palavras-chave: Agrupamento ecológico de espécies; ingresso; mortalidade.
\end{abstract}

\begin{abstract}
Effect of logging and thinning intensities in the species ecological grouping. This study was conducted with data from Projeto Bom Manejo (Embrapa-CPATU/CIFOR/ITTO, Jari area, Vitória do Jari - AP. The statistical design was in random blocks. The applied treatments are combinations of three logging intensities $(15 \%, 25 \%$ and $35 \%)$, followed by four levels of original basal area reduction $(0 \%, 30 \%, 50 \%$ and $70 \%)$. There were 40 permanent sample plots (36-logged and 4unlogged). The stand was measured in 1984, logged in 1985 and remeasured in 1986, 1988, 1990, 1994, 1996 and 2004 (trees with $\mathrm{dbh} \geq 20 \mathrm{~cm}$ ). The process for grouping species involved three stages: (1) clustering analysis to make the groups using the most populous species; (2) discriminant analysis to add the less populous species to the existing groups; and (3) subjective stage where species with little or no data were assigned into the existent groups. The characteristic variables of each species in the cluster analysis were: Periodic annual increment in diameter and the 95-percentile point in the DBH frequency distribution. It was concluded that both logging of commercial trees species and the application of thinnings stimulated the growth of trees of the plants already established, and different from expected, did not favor the growth of fast growing pioneer species.

Keywords: Species ecological grouping; ingrowth, mortality.
\end{abstract}

\section{INTRODUÇÃO}

Estima-se que a área coberta com Floresta Densa de Terra Firme na bacia amazônica brasileira foi de $3.303 .000 \mathrm{~km}^{2}$ (BRAGA, 1979). A diversidade de espécies arbóreas existentes nos diferentes tipos de floresta é alta. Em uma área de 500 ha de floresta de terra firme, na região de Manaus, foram identificadas 1077 espécies de árvores (RIBEIRO et al., 1999). Na mesma região, em um inventário 
botânico de 70 ha, foram encontradas 698 espécies arbóreas (DAP $\geq 10 \mathrm{~cm}$ ) pertencentes a 53 famílias (RANKIN DE MERONA et al., 1992). No total, estima-se que existem 12.000 espécies arbóreas na Amazônia (LLERAS; LEITE, 2005). Uma série dessas espécies é explorada comercialmente através da extração seletiva, devido ao seu valor madeireiro, sendo que, conforme a localidade, o número pode variar de 38 a 60 espécies (HIGUCHI et al., 1985) ou atingir números bem elevados, como, por exemplo, 157 (SILVA, 1989), quando são incluídas espécies com uso potencial de comercialização.

A elevada diversidade de espécies arbóreas nas florestas tropicais úmidas representa um obstáculo para as análises ecológicas. A complexidade pode ser convenientemente simplificada através da definição de grupos funcionais de espécies cujos membros partilham características funcionais que sejam úteis na determinação da estrutura e da composição da floresta, assim como para prover as respostas da vegetação quando submetidas a diferentes regimes de distúrbios (SWAINE; WHITMORE, 1988).

Métodos de agrupamento de espécies são essenciais no desenvolvimento de planos de manejo e na projeção do crescimento em florestas tropicais. Isso se deve ao fato de existir um número muito baixo de espécies que podem ser modeladas individualmente, sendo que as espécies de interesse comercial são representadas por poucos indivíduos.

No manejo florestal, o agrupamento de espécies poderia estar baseado em características comerciais ou de mercado das espécies. Entretanto, essas características não são muito apropriadas para estudo de crescimento, uma vez que classificações econômicas não são correlacionadas com o crescimento ou comportamento ecológico das espécies (VANCLAY, 1994).

Em estudos de crescimento e produção nos trópicos, vários métodos foram empregados na formação dos grupos. Por exemplo, Vanclay (1991) utilizou o teste F para agrupar funções com diferenças não significativas. Geralmente, a análise de cluster tem sido usada com base no incremento em diâmetro e algum outro parâmetro para se ter uma ordenação em pelo menos duas dimensões (ALDER, 2002; PHILLIPS et al., 2002, 2004). Outros parâmetros incluídos foram: diâmetro máximo (ALDER, 1995), diâmetro médio (EBA'A ATYI, 1997), altura (FINEGAN et al., 1999), incremento em sucessivas medidas (ATTA-BOATINGA; MOSER, 1998; PHILLIPS et al., 2002 e 2004), mortalidade, tamanho e escala comercial (ALDER; SILVA, 2000).

Técnicas puramente estatísticas não são ideais. Há escolhas subjetivas que podem influenciar os resultados e existem muitas espécies representadas por uma ou duas observações, o que reduz a efetividade e funcionalidade dos grupos formados. Para evitar esse problema, grupos funcionais baseados em características ecológicas têm sido usados em alguns estudos.

Budowski (1965) sugeriu a classificação em quatro grupos: pioneiras, secundárias, secundárias tardias e clímax. Hartshorn (1980), baseado na regeneração natural, dividiu as espécies em tolerantes e intolerantes à sombra. Também apenas dois grupos foram propostos por Swaine e Whitmore (1988), separando as pioneiras das não-pioneiras (ou clímax). Denslow (1980) distinguiu especialistas de subbosque, de clareira pequena e de clareira grande.

Neste trabalho, estudou-se o efeito de diferentes níveis de exploração e tratamentos silviculturais na formação de agrupamentos ecológicos e funcionais de espécies. Buscou-se uma classificação nãosubjetiva, com base no incremento em diâmetro e no percentual $95 \%$ da distribuição de freqüência cumulativa dos diâmetros. Definiu-se a taxa de crescimento média para cada grupo ecológico e identificaram-se as espécies dominantes de cada grupo.

\section{MATERIAL E MÉTODO}

\section{Localização e caracterização da área}

Este estudo foi conduzido com dados do Projeto Bom Manejo (Embrapa Amazônia Ocidental/CIFOR/ITTO). O experimento foi iniciado em 1983, em uma área de 500 ha da Companhia Florestal Monte Dourado (Jari), na localidade Morro do Felipe, Vitória do Jari (AP), entre as coordenadas $52^{0} 20^{\prime \prime} \mathrm{W}$ e $00^{0} 55^{\prime \prime S}$, a uma altitude de aproximadamente $150 \mathrm{~m}$. O clima nessa região é do tipo Ami, pela classificação de Köppen. A precipitação média anual alcança $2.234 \mathrm{~mm}$. A temperatura média anual é de $25,8{ }^{0} \mathrm{C}$ e a vegetação é do tipo Floresta Ombrófila Densa. Os solos são do tipo Latossolo Amarelo Distrófico, com textura argilosa pesada. 


\section{Delineamento experimental}

$\mathrm{O}$ delineamento foi em blocos ao acaso com três repetições. As alternativas de manejo testadas foram combinações de intensidades de exploração com intensidades de desbastes. As intensidades de exploração $(15 \%$ - intensidade leve, $25 \%$ - intensidade média e $35 \%$ - intensidade pesada) foram representadas por porcentagens do volume extraído em relação ao volume total da floresta, a partir do diâmetro à altura do peito (dap) de $60 \mathrm{~cm}$. Elas foram combinadas com quatro tipos de intensidades de desbastes, o desbaste sistemático e o desbaste seletivo nas intensidades de $0 \%, 30 \%, 50 \%$ e $70 \%$ de redução da área basal original (Tabela 1 e Figura 1).

Tabela 1. Descrição dos tratamentos.

Table 1. Description of the treatments.

\begin{tabular}{|c|c|}
\hline Tratamento & Descrição \\
\hline $\mathrm{T}_{1}$ & $\begin{array}{l}\text { Exploração de } 15 \% \text { do volume total das árvores de dap }>60 \mathrm{~cm} \text {, sem redução da área basal após a } \\
\text { exploração. }\end{array}$ \\
\hline $\mathrm{T}_{2}$ & $\begin{array}{l}\text { Exploração de } 15 \% \text { do volume total das árvores de dap }>60 \mathrm{~cm} \text {, com redução de } 30 \% \text { da área basal } \\
\text { original através de tratos silviculturais. }\end{array}$ \\
\hline $\mathrm{T}_{3}$ & $\begin{array}{l}\text { Exploração de } 15 \% \text { do volume total das árvores de dap }>60 \mathrm{~cm} \text {, com redução de } 50 \% \text { da área basal } \\
\text { original através de tratos silviculturais. }\end{array}$ \\
\hline $\mathrm{T}_{4}$ & $\begin{array}{l}\text { Exploração de } 15 \% \text { do volume total das árvores de dap }>60 \mathrm{~cm} \text {, com redução de } 70 \% \text { da área basal } \\
\text { original através de tratos silviculturais. }\end{array}$ \\
\hline $\mathrm{T}_{5}$ & $\begin{array}{l}\text { Exploração de } 25 \% \text { do volume total das árvores de dap }>60 \mathrm{~cm} \text {, sem redução da área basal após a } \\
\text { exploração. }\end{array}$ \\
\hline $\mathrm{T}_{6}$ & $\begin{array}{l}\text { Exploração de } 25 \% \text { do volume total das árvores de dap }>60 \mathrm{~cm} \text {, com redução de } 30 \% \text { da área basal } \\
\text { original através de tratos silviculturais. }\end{array}$ \\
\hline $\mathrm{T}_{7}$ & $\begin{array}{l}\text { Exploração de } 25 \% \text { do volume total das árvores de dap }>60 \mathrm{~cm} \text {, com redução de } 50 \% \text { da área basal } \\
\text { original através de tratos silviculturais. }\end{array}$ \\
\hline $\mathrm{T}_{8}$ & $\begin{array}{l}\text { Exploração de } 25 \% \text { do volume total das árvores de dap }>60 \mathrm{~cm} \text {, com redução de } 70 \% \text { da área basal } \\
\text { original através de tratos silviculturais. }\end{array}$ \\
\hline $\mathrm{T}_{9}$ & $\begin{array}{l}\text { Exploração de } 35 \% \text { do volume total das árvores de dap }>60 \mathrm{~cm} \text {, sem redução da área basal após a } \\
\text { exploraça. }\end{array}$ \\
\hline $\mathrm{T}_{10}$ & $\begin{array}{l}\text { Exploração de } 35 \% \text { do volume total das árvores de dap }>60 \mathrm{~cm} \text {, com redução de } 30 \% \text { da área basal } \\
\text { original através de tratos silviculturais. }\end{array}$ \\
\hline $\mathrm{T}_{11}$ & $\begin{array}{l}\text { Exploração de } 35 \% \text { do volume total das árvores de dap }>60 \mathrm{~cm} \text {, com redução de } 50 \% \text { da área basal } \\
\text { original através de tratos silviculturais. }\end{array}$ \\
\hline $\mathrm{T}_{12}$ & $\begin{array}{l}\text { Exploração de } 35 \% \text { do volume total das árvores de dap }>60 \mathrm{~cm} \text {, com redução de } 70 \% \text { da área basal } \\
\text { original através de tratos silviculturais. }\end{array}$ \\
\hline
\end{tabular}

\section{Monitoramento da floresta}

$\mathrm{Na}$ área experimental estão locadas 40 parcelas permanentes de um hectare. Trinta e seis em área explorada e quatro em área não-explorada. A primeira medição foi realizada em 1984, a exploração em 1985 e as remedições em 1986, 1988, 1990, 1994, 1996 e 2004 (Figura 1). Todas as árvores com diâmetro maior ou igual a $20 \mathrm{~cm}$ foram medidas. A metodologia empregada foi desenvolvida e disponibilizada por Silva e Lopes (1984).

\section{Agrupamento ecológico de espécies}

O processo para agrupamento das espécies envolveu três estágios: Análise de Cluster (espécies populosas $-\mathrm{n}^{0}$ de indivíduos $\left.\geq 50\right)$, Análise Discriminante $\left(10 \leq \mathrm{n}^{0}\right.$ de indivíduos $\left.<50\right)$ e, Método Subjetivo $\left(\mathrm{n}^{0}\right.$ de indivíduos $\left.<10\right)$.

\section{Análise de Cluster: espécies mais populosas}

As variáveis características de cada espécie na Análise de Cluster foram: taxa média de crescimento e percentil 95\% da distribuição de freqüência cumulativa dos diâmetros (como um índice do comportamento da mortalidade). As taxas de crescimento foram avaliadas usando cada um dos intervalos de medição: 1984-1986, 1986-1988, 1988-1990, 1990-1994, 1994-1996, 1996-2004. 
Análise Discriminante: espécies menos populosas

$\mathrm{Na}$ abordagem usada, foram tomadas as espécies com pelo menos 10 e menos de 50 árvores nos dados, usando-se o ponto percentil $95 \%$ na distribuição cumulativa de dap, e a taxa média de crescimento como características para acrescentar as espécies aos grupos existentes (Análise de Cluster).

Método Subjetivo: acrescentando os grupos taxonômicos restantes aos grupos formados

As espécies que restaram foram designadas aos grupos existentes através de características botânicas ou informações disponíveis na literatura, tais como incremento, diâmetro máximo e taxa de crescimento.

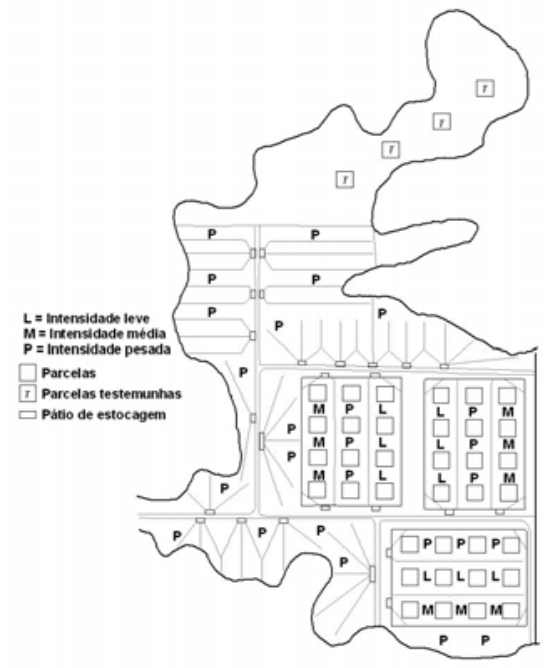

Figura 1. Croqui do experimento (L: intensidade leve; M: intensidade média; P: intensidade pesada).

Figure 1. Croquis of the experiment (L: light intensity; M: mean intensity; P: heavy intensity).

\section{RESULTADOS E DISCUSSÃO}

\section{Análise de cluster: espécies mais populosas}

No primeiro estágio de agrupamento usando espécies com pelo menos 50 árvores, ocorreram 77 espécies, representando $64,97 \%$ do total de árvores amostradas (Tabela 2). Nesse estágio, vinte e cinco espécies não apresentaram incremento periódico anual e não puderam ser utilizadas. Assim, só 52 espécies foram usadas nessa fase.

Tabela 2. Nível populacional por espécie na Floresta da Jari.

Table 2. Minimum number of trees in a taxon for the Jari permanent sample plots.

\begin{tabular}{lccc}
\hline $\mathbf{N}^{\mathbf{0}}$ de espécies & $\mathbf{N}^{\mathbf{0}}$ mínimo de árvores & $\begin{array}{c}\mathbf{N}^{\mathbf{0}} \text { de árvores } \\
(\mathbf{N})\end{array}$ & $\mathbf{N}^{\mathbf{0}}$ de árvores acumuladas $(\%)$ \\
\hline 25 & 100 & 4730 & 47,51 \\
77 & 50 & 6468 & 64,97 \\
170 & 10 & 9188 & 92,30 \\
427 & 1 & 9955 & 100,00 \\
\hline
\end{tabular}

O processo de agrupamento exige que o usuário decida, com antecedência, quantos grupos deve haver. A escolha do número de grupos é subjetiva, devendo ser um equilíbrio entre ter mais dados por grupo e mais grupos para representar a variação dentro da floresta. Com representações visuais, usando os valores das variáveis características, é possível prover julgamento subjetivo sobre a utilidade do agrupamento. Isso é relativamente fácil com 2 variáveis, porém, nenhum julgamento subjetivo foi empreendido nesta análise. $\mathrm{O}$ total de 5 grupos foi tomado com base no equilíbrio acima e na facilidade 
de nominação desses grupos (Tabela 3). Dez agrupamentos foram definidos nos estudos prévios de Phillips et al. (2001, 2004). Entretanto, nos dez grupos, ficou difícil separar as características de crescimento e ecológicas das espécies.

Os 52 grupos taxonômicos que tinham pelo menos 50 árvores registradas nos dados, usados para fazer os agrupamentos, responderam por 25.237 medidas de crescimento (Tabela 3 ).

Tabela 3. Resumo das características dos grupos de espécies.

Table 3. A summary of the characteristics of the species groups.

\begin{tabular}{|c|c|c|c|c|c|c|c|c|c|}
\hline $\mathbf{G}$ & Nome & $\mathbf{N}_{\mathrm{t}}$ & $\mathbf{N}_{t}(\%)$ & Ns & $\mathbf{N}_{I P A}$ & $P_{95}$ & $s P_{95}$ & $\overline{I P A}$ & SIPA \\
\hline 1 & $\begin{array}{l}\text { Crescimento muito lento, dossel } \\
\text { inferior }\end{array}$ & 2080 & 32,16 & 23 & 7251 & 41,70 & 0,0828 & 0,22 & 0,0033 \\
\hline 2 & Crescimento lento, dossel médio & 2995 & 46,30 & 18 & 13149 & 59,70 & 0,1128 & 0,25 & 0,0024 \\
\hline 3 & $\begin{array}{l}\text { Crescimento moderado, emergentes } \\
\text { - climácicas }\end{array}$ & 854 & 13,20 & 7 & 3559 & 92,20 & 0,3782 & 0,34 & 0,0058 \\
\hline 4 & Crescimento rápido, dossel médio & 435 & 6,73 & 3 & 957 & 67,40 & 0,5082 & 0,82 & 0,0203 \\
\hline 5 & $\begin{array}{l}\text { Crescimento muito rápido, dossel } \\
\text { superior }\end{array}$ & 104 & 1,61 & 1 & 321 & 74,00 & 0,9255 & 1,41 & 0,0422 \\
\hline Total & & 6468 & 100 & 52 & 25.237 & & & & \\
\hline
\end{tabular}

G: número do grupo; $\mathrm{P}_{95}$ : percentil 95 da distribuição acumulada de diâmetro $(\mathrm{cm})$; IPA: incremento periódico anual em diâmetro $\left(\mathrm{cm} . \mathrm{ano}^{-1}\right) ; \mathrm{N}_{\mathrm{t}}$ : número de árvores; $\mathrm{N}_{\mathrm{s}}$ : número de espécies, em cada grupo; $\mathrm{sIPA}$ e $\mathrm{sP}_{95}$ : desvios padrões.

\section{Análise discriminante: espécies menos populosas}

As espécies com pelo menos 10 observações e menos que cinqüenta foram acrescentadas aos grupos existentes usando-se as mesmas variáveis do agrupamento das espécies mais populosas, em uma análise de discriminante.

Os 116 grupos taxonômicos (espécies) que tinham pelo menos 10 e menos de 50 árvores nos dados, que foram acrescentados aos grupos existentes, responderam por 10.016 medidas de crescimento. Depois desse processo, 35.253 observações de crescimento puderam ser designadas a um dos 5 agrupamentos de espécies, equivalente a 168 grupos taxonômicos (Figura 2).

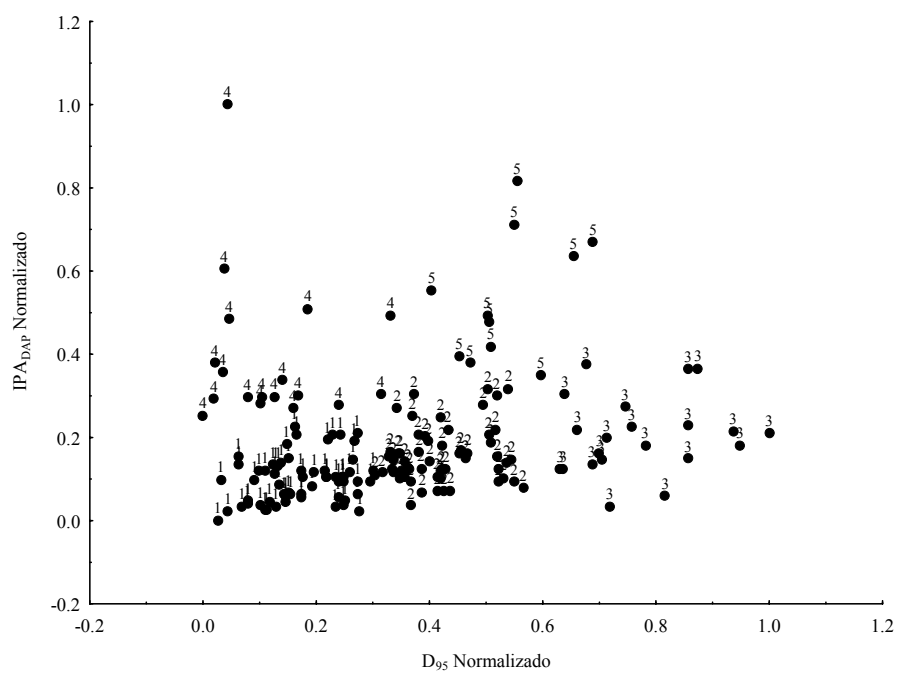

Figura 2. Distribuição das 168 espécies nos cinco agrupamentos formados, após a análise discriminante. Figure 2. Distribution of the 168 species in the five formed groupings, after the discriminate analysis.

\section{Estágio subjetivo}

As espécies restantes, não agrupadas, foram acrescentadas aos grupos existentes usando uma segunda análise discriminante associada à variável incremento médio anual. Esses dados foram usados na ausência de informações alternativas. 
Trinta e seis espécies, noventa e seis árvores, representadas por somente uma medida de diâmetro durante os inventários, não puderam ser agrupadas pelo segundo discriminante. Essas espécies foram adicionadas aos grupos existentes com base em informações disponíveis na literatura, descritas anteriormente.

Os dados compreenderam 37.855 medidas de crescimento. As 219 espécies com menos de dez árvores que foram adicionadas aos grupos existentes contribuíram com 2.602 taxas de crescimento e 698 árvores. Após esse processo 37.855 taxas de crescimento foram encontradas nos cinco agrupamentos, 381 espécies (Tabela 4).

Tabela 4. Descrição dos cinco grupos resultantes do processo de agrupamento. Table 4. Description of the five groups resulting from the grouping process.

\begin{tabular}{llrrrrrrrr}
\hline $\mathbf{G}$ & \multicolumn{1}{c}{ Nome } & $\mathbf{N}_{\mathbf{t}}$ & $\mathbf{N}_{\mathbf{t}} \mathbf{( \% )}$ & $\mathbf{N s}$ & $\mathbf{N}_{\boldsymbol{I P A}}$ & $\boldsymbol{P}_{\mathbf{9 5}}$ & $\boldsymbol{s}_{\mathbf{9 5}}$ & $\overline{I P A}$ & $\boldsymbol{s I P A}$ \\
\hline \multirow{2}{*}{1} & Crescimento muito lento, dossel & & & & & & & & \\
2 & inferior & 3125 & 31,70 & 136 & 11531 & 42,70 & 0,0754 & 0,20 & 0,0024 \\
& Crescimento lento, dossel médio & 3918 & 39,74 & 68 & 17105 & 60,00 & 0,1000 & 0,27 & 0,0022 \\
3 & Crescimento moderado, emergentes - & & & & & & & & \\
& climácicas & 1266 & 12,84 & 52 & 5350 & 91,10 & 0,3162 & 0,34 & 0,0049 \\
4 & Crescimento rápido, dossel médio & 995 & 10,09 & 96 & 2145 & 71,40 & 0,3999 & 0,54 & 0,0105 \\
5 & Crescimento muito rápido, dossel & & & & & & & & \\
& superior & 555 & 5,63 & 29 & 1724 & 72,50 & 0,4164 & 0,95 & 0,0167 \\
\hline Total & & 9859 & 100,00 & 381 & 37.855 & & & & \\
\hline
\end{tabular}

G: número do grupo; $\mathrm{P}_{95}$ : percentil 95 da distribuição acumulada de diâmetro $(\mathrm{cm})$; IPA: incremento periódico anual em diâmetro $\left(\mathrm{cm} . \mathrm{ano}^{-1}\right) ; \mathrm{N}_{\mathrm{t}}$ : número de árvores; $\mathrm{N}_{\mathrm{s}}$ : número de espécies, em cada grupo; $\mathrm{sIPA}$ e $\mathrm{sP}_{95}$ : desvios padrões.

\section{Espécies dominantes em cada grupo}

Os resultados corresponderam às expectativas, principalmente para os grupos 3 e 5 , com respeito às espécies que os formam, dominados por espécies de valor comercial, de crescimento moderado, clímax a crescimento muito rápido (Tabela 5).

O grupo 4 é composto, principalmente, de espécies pioneiras de rápido crescimento e vida curta. A espécie Jacaranda copaia, que pode se tornar muito mais comum em paisagens fortemente perturbadas por atividade humana ou fenômenos naturais (FINEGAN, 1992), é considerada pioneira de vida longa e de rápido crescimento (PHILLIPS et al., 2002). Aqui, essa espécie foi considerada de crescimento muito lento e pertencente ao dossel inferior, sugerindo que alguns ajustes precisam ser feitos para algumas espécies (Tabela 5).

Grupo 1: Protium sagotianum, Eschweilera coriacea, Eschweilera amazonica, Drypetes variabilis, Protium opacum, Anaxagorea dolichocarpa, Rinorea guianensis, Jacaranda copaia, Inga spp., Aniba albescens, Pithecellobium cochleatum, Duguetia surinamensis, Guatteria poeppigiana, Ocotea dissimilis, Eschweilera fracta, Diplotropis purpurea, Cecropia obtusa, Couratari guianensis, Eschweilera subglandulosa, Lecythis usitata, Cordia goeldiana, Hymenaea intermedia, Vataireopsis speciosa, Calophyllum brasiliensis, Maquira sclerophylla e Virola calophylla.

Grupo 2: Geissospermum sericeum, Eschweilera jurunensis, Minquartia guianensis, Micropholis guianensis, Ocotea douradensis, Virola michelli, Lecythis poitequi, Mouriri callocarpa, Nectandra myriantha, Gustavia hexapetala, Syzygiopsis pachycarpa, Pouteria macrocarpa, Mezilaurus lindaviana, Chimarrhis turbinata, Prieurella preurii, Trattinickia rhoifolia, Chamaecrista bahiae, Apeiba burchellii, Symphonia globulifera, Tabebuia serratifolia, Carapa guianensis, Dialium guianensis, Marmaroxylon racemosum, Licaria canella, Dipteryx magnifica, Qualea paraensis, Aniba fragans, Persea jariensis e Aniba canellila.

Grupo 3: Manilkara huberi, Goupia glabra, Syzygiopsis oppositifolia, Manilkara bidentata, Dipteryx odorata, Pseudopiptadenia psilostachya, Terminalia argentea, Endopleura uch, Sloanea obtusa, Brosimum acutifolium, Hymenaea courbaril, Bowdichia nitida, Swartzia racemosa, Terminalia amazônica, Aspidosperma spruceana, Dimorphandra gardneriana e Copaifera martii.

Grupo 4: Cecropia sciadophylla, Inga paraensis, Inga heterophylla, Xylopia nitida, Pourouma vilosa, Simaruba amara, Lecythis lúrida, Ormosia flava, Tetragastris altíssima, Swartzia polyphylla, Enterolobium schomburgkii, Martiodendron parviflorum, Miconia surinamensis, Malouetia duckey, Emmotum fagifolium, Clarisia racemosa, Inga capitata, Inga rubiginosa, Pouteria crassifolia, 
Aspidosperma carapanauba, Hymenolobium excelsum, Dinizia excelsa, Tachigalia paraensis e Bertholletia excelsa.

Grupo 5: Sclerolobium tinctorium, Tachigalia myrmecophyla, Inga alba, Qualea albiflora, Parkia ulei, Tapirira guianensis, Parkia decussata, Macoubea guianensis, Parkia oppositifolia, Sclerolobium melanocarpum, Stryphnodendron pulcherrimum, Osteophloeum platyspermum, Cassia spruceana, Protium hostmannii, Trattinickia burserifolia, Dimorphandra surtifolia, Sclerolobium paraense e Ocotea sprucei.

A relação completa das espécies por agrupamento ecológico, o incremento periódico médio anual e o processo de agrupamento é mostrado na tabela 5.

Tabela 5. Relação de espécies e famílias existentes na área experimental da Jari (N: número de árvore; IPA: incremento periódico em diâmetro $\left(\mathrm{cm}^{-a n o}{ }^{-1}\right) ; \mathrm{P}_{95}(\mathrm{~cm})$ : percentil $95 \%$ da distribuição cumulativa dos diâmetros; G: grupo ecológico de espécies; D: discriminação (0 - cluster; 1 discriminante; 3 - subjetivo; 4 - manual); NI: não identificada.

Table 5. List of species and families in the experimental area of Jari (N: number of trees; IPA: periodic annual increment in diameter $\left(\mathrm{cm}^{-a n 0^{-1}}\right) ; \mathrm{P}_{95}(\mathrm{~cm})$ : percentile $95 \%$ of the cumulative distribution of the diameter; G: Species ecological group; D: discrimination (0 - Cluster; 1 Discriminant; 3 - Subjective; 4 - Manual); NI: not identified.

\begin{tabular}{|c|c|c|c|c|c|c|}
\hline Família & Nome Científico & $\mathbf{N}$ & IPA & $\mathbf{P}_{95}$ & $\mathbf{G}$ & D \\
\hline \multirow[t]{8}{*}{ ANACARDIACEAE } & Anacardium giganteum & 10 & 0,54 & 70,50 & 2 & 1 \\
\hline & Anacardium spruceanum & 5 & 0,34 & 90,80 & 4 & 2 \\
\hline & Astronium gracile & 1 & 0,07 & 97,10 & 1 & 2 \\
\hline & Astronium obliquum & 10 & 0,35 & 61,40 & 2 & 1 \\
\hline & Tapirira guianensis. & 46 & 0,68 & 66 & 5 & 1 \\
\hline & Tapirira spp & 17 & 0,57 & 69,10 & 2 & 1 \\
\hline & Thyrsodium guianensis & 12 & 0,26 & 33,70 & 1 & 1 \\
\hline & Thyrsodium spruceanum & 2 & 0,07 & 22,50 & 1 & 2 \\
\hline \multirow[t]{13}{*}{ ANNONACEAE } & Anaxagorea dolichocarpa & 101 & 0,21 & 24 & 1 & 0 \\
\hline & Annona sericea & 1 & & & 1 & 4 \\
\hline & Bocageopsis multiflora & 4 & 0,24 & 36,80 & 3 & 2 \\
\hline & Duguetia cauliflora & 2 & 0,62 & 21 & 4 & 2 \\
\hline & Duguetia surinamensis & 44 & 0,21 & 29,70 & 1 & 1 \\
\hline & Guatteria amazonica & 1 & & & 1 & 4 \\
\hline & Guatteria poeppigiana & 48 & 0,39 & 36,80 & 1 & 1 \\
\hline & Guatteriopsis spp & 5 & 0,24 & 25,30 & 4 & 2 \\
\hline & $\mathrm{Ni}$ & 9 & 0,12 & 35,80 & 1 & 2 \\
\hline & Onychopetalum amazonicum & 1 & 0,22 & 29,30 & 3 & 2 \\
\hline & Rollinia fendleri & 1 & 0,06 & 69,30 & 1 & 2 \\
\hline & Xylopia spp & 2 & 0,76 & 21,70 & 5 & 2 \\
\hline & Xylopia nitida & 26 & 0,49 & 36,20 & 4 & 1 \\
\hline \multirow[t]{16}{*}{ APOCYNACEAE } & Aspidosperma atanum & 38 & 0,20 & 49,30 & 1 & 1 \\
\hline & Aspidosperma carapanauba & 7 & 0,70 & 74,50 & 4 & 2 \\
\hline & Aspidosperma centrale & 4 & 0 & 45,50 & 1 & 2 \\
\hline & Aspidosperma desmanthum & 1 & 0,06 & 27,80 & 1 & 2 \\
\hline & Aspidosperma macrocarpum & 1 & 0,14 & 57,60 & 1 & 2 \\
\hline & Aspidosperma megalocarpum & 1 & & & 1 & 4 \\
\hline & Aspidosperma oblongum & 4 & 0,48 & 22,20 & 4 & 2 \\
\hline & Aspidosperma paraensis & 8 & 0,38 & 101,10 & 4 & 2 \\
\hline & Aspidosperma sandwithianum & 1 & & & 1 & 4 \\
\hline & Aspidosperma spp & 51 & 0,40 & 110,40 & 3 & 0 \\
\hline & Aspidosperma spruceana & 12 & 0,42 & 102,80 & 3 & 1 \\
\hline & Couma guianensis & 1 & & & 1 & 4 \\
\hline & Geissospermum sericeum & 502 & 0,21 & 54,30 & 2 & 0 \\
\hline & Himatanthus sucuuba & 5 & 0,39 & 27,40 & 4 & 2 \\
\hline & Macoubea guianensis & 15 & 0,87 & 68,90 & 5 & 1 \\
\hline & Malouetia duckey & 7 & 0,36 & 48,30 & 4 & 2 \\
\hline AQUIFOLIACEAE & Ilex martiniana & 3 & 0,22 & 37,60 & 3 & 2 \\
\hline ARALIACEAE & Didymopanax morototoni & 4 & & & 1 & 4 \\
\hline \multirow[t]{3}{*}{ BIGNONIACEAE } & Jacaranda caucana & 11 & 0,25 & 61,30 & 2 & 1 \\
\hline & Jacaranda copaia & 78 & 0,38 & 42,90 & 1 & 0 \\
\hline & Tabebuia serratifolia & 28 & 0,10 & 56,10 & 2 & 1 \\
\hline \multirow{2}{*}{ BOMBACACEAE } & Bombacopsis nervosa & 21 & 0,42 & 36,40 & 1 & 1 \\
\hline & Eriotheca globosa & 2 & 0,16 & 29,70 & 2 & 2 \\
\hline
\end{tabular}

FLORESTA, Curitiba, PR, v. 38, n. 1, jan./mar. 2008. 


\begin{tabular}{|c|c|c|c|c|c|c|}
\hline Família & Nome Científico & $\mathbf{N}$ & IPA & $\mathbf{P}_{95}$ & $\mathbf{G}$ & $\mathbf{D}$ \\
\hline & Matisia paraensis & 1 & 0,71 & 21,20 & 5 & 2 \\
\hline & $\mathrm{Ni}$ & 1 & 0,17 & 24,20 & 2 & 2 \\
\hline & Pachira aquatica & 2 & 0,47 & 21,90 & 4 & 2 \\
\hline & Pachira sp & 1 & 0,51 & 23,90 & 4 & 2 \\
\hline & Pseudobombax munguba & 1 & 0,26 & 20,90 & 3 & 2 \\
\hline & Pseudobombax spp & 1 & 1,19 & 21,50 & 5 & 2 \\
\hline & Quaribea guianensis & 2 & 0,23 & 26,70 & 3 & 2 \\
\hline \multirow{3}{*}{ BORAGINACEAE } & Cordia goeldiana & 6 & 0,05 & 49,60 & 1 & 2 \\
\hline & Cordia scabrifolia & 8 & 0,15 & 24,70 & 1 & 2 \\
\hline & Cordia spp & 3 & 0,02 & 20,80 & 1 & 2 \\
\hline \multirow[t]{15}{*}{ BURSERACEAE } & Dacryodes nitens & 6 & 0,18 & 39,20 & 2 & 2 \\
\hline & $\mathrm{Ni}$ & 19 & 0,11 & 30,70 & 1 & 1 \\
\hline & Paraprotium amazonicum & 35 & 0,27 & 27,10 & 1 & 1 \\
\hline & Protium decandrum & 55 & 0,24 & 30,50 & 1 & 0 \\
\hline & Protium hostmannii & 1 & 0,88 & 28,20 & 5 & 2 \\
\hline & Protium krukaffii & 5 & 0,54 & 20,70 & 4 & 2 \\
\hline & Protium opacum & 83 & 0,26 & 33,30 & 1 & 0 \\
\hline & Protium pallidum & 4 & 0,38 & 24 & 4 & 2 \\
\hline & Protium robustum & 1 & & & 1 & 4 \\
\hline & Protium sagotianum & 175 & 0,23 & 39,80 & 1 & 0 \\
\hline & Protium sp & 1 & 0,50 & 20,30 & 4 & 2 \\
\hline & Tetragastris altissima & 8 & 0,47 & 58 & 4 & 2 \\
\hline & Tetragastris paraensis & 44 & 0,15 & 37,60 & 1 & 1 \\
\hline & Trattinickia burserifolia & 1 & 0,89 & 33,10 & 5 & 2 \\
\hline & Trattinickia rhoifolia & 36 & 0,36 & 69,50 & 2 & 1 \\
\hline \multirow[t]{34}{*}{ CAESALPINIACEAE } & Batesia floribunda & 2 & 0,74 & 51,20 & 4 & 2 \\
\hline & Bocoa alterna & 1 & 0,08 & 41,80 & 1 & 2 \\
\hline & Cassia spruceana & 5 & 0,79 & 66,50 & 5 & 2 \\
\hline & Chamaecrista bahiae & 44 & 0,26 & 55,60 & 2 & 1 \\
\hline & Copaifera martii & 10 & 0,50 & 92,20 & 3 & 1 \\
\hline & Cynometra spruceanum & 3 & 0 & 21 & 1 & 2 \\
\hline & Dialium guianensis & 24 & 0,20 & 73,50 & 2 & 1 \\
\hline & Dimorphandra gardneriana & 10 & 0,67 & 85,60 & 3 & 1 \\
\hline & Dimorphandra macrostachya & 1 & & & 3 & 4 \\
\hline & Dimorphandra surtifolia & 1 & 0,72 & 21,20 & 5 & 2 \\
\hline & Elizabetha bicolor & 12 & 0,22 & 44,50 & 1 & 1 \\
\hline & Hymenaea courbaril & 40 & 0,39 & 116,50 & 3 & 1 \\
\hline & Hymenaea intermédia & 4 & 0,10 & 59,10 & 1 & 2 \\
\hline & Macrolobium acaciaefolim & 1 & 0,01 & 33,70 & 1 & 2 \\
\hline & Martiodendron parviflorum & 6 & 0,47 & 99 & 4 & 2 \\
\hline & $\mathrm{Ni}$ & 3 & 0,35 & 30,30 & 4 & 2 \\
\hline & Peltogyne paradoxa & 4 & 0,35 & 57,50 & 4 & 2 \\
\hline & Recordoxylon stenopetalum & 1 & 0,07 & 57 & 1 & 2 \\
\hline & Sclerolobium melanocarpum & 11 & 1,23 & 73,50 & 5 & 1 \\
\hline & Sclerolobium melinonii & 8 & & & 5 & 4 \\
\hline & Sclerolobium paraense & 6 & 1,80 & 23,10 & 5 & 2 \\
\hline & Sclerolobium spp & 6 & 1,15 & 82,80 & 5 & 2 \\
\hline & Sclerolobium tinctorium & 145 & 0,84 & 69,30 & 5 & 0 \\
\hline & Swartzia grandiflora & 1 & 0,23 & 20,80 & 3 & 2 \\
\hline & Swartzia panacoco & 4 & 0,26 & 42,30 & 3 & 2 \\
\hline & Swartzia polyphylla & 9 & 0,56 & 81,60 & 4 & 2 \\
\hline & Swartzia racemosa & 16 & 0,29 & 102,80 & 3 & 1 \\
\hline & Tachigalia alba & 1 & & & 5 & 4 \\
\hline & Tachigalia myrmecophyla & 104 & 1,41 & 74 & 5 & 0 \\
\hline & Tachigalia paraensis & 11 & 1,72 & 25,10 & 4 & 1 \\
\hline & Tachigalia spp & 2 & 0,92 & 21,60 & 5 & 2 \\
\hline & Tachigalia spruceana & 2 & & & 5 & 4 \\
\hline & Vouacapoua americana & 6 & 0,38 & 28,70 & 4 & 2 \\
\hline & Zizyphus tacaiunensis & 1 & 0,46 & 21,30 & 4 & 2 \\
\hline \multirow[t]{2}{*}{ CARYOCARACEAE } & Caryocar glabrum & 1 & 0,83 & 24,10 & 5 & 2 \\
\hline & Caryocar villosum. & 5 & 0,30 & 93 & 4 & 2 \\
\hline \multirow{4}{*}{ CELASTRACEAE } & Goupia glabra & 225 & 0,27 & 86,60 & 3 & 0 \\
\hline & Maytenus floribunda & 110 & 0,14 & 37,60 & 1 & 0 \\
\hline & Maytenus myrsinoides & 3 & 0,21 & 39,70 & 3 & 2 \\
\hline & $\mathrm{Ni}$ & 1 & $-0,02$ & 43,70 & 1 & 2 \\
\hline CHRYSOBALANACEAE & Couepia guianensis & 1 & 0,15 & 34,40 & 1 & 2 \\
\hline
\end{tabular}




\begin{tabular}{|c|c|c|c|c|c|c|}
\hline Família & Nome Científico & $\mathbf{N}$ & IPA & $\mathbf{P}_{95}$ & $\mathbf{G}$ & D \\
\hline & Couepia robusta & 1 & 0,37 & 22,50 & 4 & 2 \\
\hline & Hirtella bicornis & 2 & 0,22 & 26,40 & 3 & 2 \\
\hline & Hirtella piresii & 2 & 0,18 & 25,10 & 2 & 2 \\
\hline & Hirtella spp & 9 & 0,23 & 29,50 & 3 & 2 \\
\hline & Licania heteromorpha & 6 & 0,24 & 29,60 & 3 & 2 \\
\hline & Licania impressa & 1 & 0,14 & 25,80 & 1 & 2 \\
\hline & Licania kunthiana & 4 & 0,23 & 42,30 & 3 & 2 \\
\hline & Licania latifolia & 18 & 0,32 & 52,60 & 2 & 1 \\
\hline & Licania latimorpha & 1 & & & 2 & 4 \\
\hline & Licania macrophylla & 8 & 0,29 & 58,40 & 3 & 2 \\
\hline & Licania membranacea & 4 & 0,44 & 28 & 4 & 2 \\
\hline & Licania paraensis & 9 & 0,17 & 56,60 & 1 & 2 \\
\hline & Licania robusta & 3 & 0,30 & 55,20 & 3 & 2 \\
\hline & $\mathrm{Ni}$ & 4 & 0,15 & 23 & 1 & 2 \\
\hline & Parinari excelsa & 8 & 0,26 & 56,50 & 3 & 2 \\
\hline \multirow{2}{*}{ CLUSIACEAE } & Vismia cayanensis & 1 & 0,48 & 25,50 & 4 & 2 \\
\hline & Vismia $\mathrm{sp}$ & 1 & & & 4 & 4 \\
\hline \multirow[t]{9}{*}{ COMBRETACEAE } & Buchenavia grandis & 1 & 0,28 & 59,80 & 3 & 2 \\
\hline & Buchenavia huberi & 1 & 0,30 & 63,70 & 4 & 2 \\
\hline & Buchenavia spp & 1 & 0,62 & 20,70 & 4 & 2 \\
\hline & Buchenavia parvifolia & 1 & 0,57 & 48,20 & 4 & 2 \\
\hline & $\mathrm{Ni}$ & 16 & 0,37 & 89,10 & 3 & 1 \\
\hline & Terminalia amazonica & 13 & 0,35 & 95,60 & 3 & 1 \\
\hline & Terminalia argentea & 36 & 0,35 & 111,60 & 3 & 1 \\
\hline & Terminalia spp & 2 & 0,65 & 76,40 & 4 & 2 \\
\hline & Terminalia spp & 2 & & & 3 & 4 \\
\hline CONNARACEAE & Connarus perrottettil & 2 & 0,11 & 26 & 1 & 2 \\
\hline DICHAPETALACEAE & Tapura amazonica & 1 & & & 1 & 4 \\
\hline \multirow[t]{5}{*}{ EBENACEAE } & Diospyros duckey & 2 & 0,04 & 30 & 1 & 2 \\
\hline & Diospyros guianensis & 1 & & & 1 & 4 \\
\hline & Diospyros praetermissa & 65 & 0,15 & 34,70 & 1 & 0 \\
\hline & Diospyros santaremnensis & 37 & 0,29 & 35,40 & 1 & 1 \\
\hline & Diospyros spp & 15 & 0,15 & 35,40 & 1 & 1 \\
\hline \multirow[t]{5}{*}{ ELAEOCARPACEAE } & Sloanea grandis & 14 & 0,20 & 44,60 & 1 & 1 \\
\hline & Sloanea guianensis & 1 & 0,30 & 22,50 & 4 & 2 \\
\hline & Sloanea spp & 6 & 0,20 & 24,80 & 3 & 2 \\
\hline & Sloanea obtusa & 40 & 0,25 & 81,30 & 3 & 1 \\
\hline & Sloanea spp & 18 & 0,20 & 56 & 2 & 1 \\
\hline \multirow[t]{8}{*}{ EUPHORBIACEAE } & Conceveiba guianensis. & 6 & 0,20 & 34,80 & 2 & 2 \\
\hline & Conceveibastrum martianum & 8 & 0,17 & 28,70 & 1 & 2 \\
\hline & Drypetes variabilis & 94 & 0,24 & 31,50 & 1 & 0 \\
\hline & Glycidendron amazonicum & 16 & 0,39 & 47 & 1 & 1 \\
\hline & Mabea caudata & 27 & 0,12 & 28,60 & 1 & 1 \\
\hline & Mabea $\mathrm{sp}$ & 1 & 0,15 & 20,60 & 1 & 2 \\
\hline & Pogonophora schomburgkiana & 21 & 0,29 & 46,30 & 1 & 1 \\
\hline & Sagotia racemosa & 6 & 0,05 & 20,50 & 1 & 2 \\
\hline \multirow{20}{*}{ FABACEAE } & Andira surinamensis & 2 & 0,31 & 29,10 & 4 & 2 \\
\hline & Bowdichia nitida & 18 & 0,10 & 89,60 & 3 & 1 \\
\hline & Dalbergia spruceana & 1 & 0,21 & 59,40 & 1 & 2 \\
\hline & Diplotropis purpurea & 7 & 0,19 & 74,20 & 1 & 2 \\
\hline & Diplotropis racemosa & 1 & 0,07 & 56,50 & 1 & 2 \\
\hline & Dipteryx magnifica & 15 & 0,25 & 62 & 2 & 1 \\
\hline & Dipteryx odorata & 58 & 0,14 & 99 & 3 & 0 \\
\hline & Dussia discolor & 1 & & & 1 & 4 \\
\hline & Hymenolobium excelsum & 6 & 0,32 & 126 & 4 & 2 \\
\hline & Hymenolobium flavum & 2 & 0,18 & 51,20 & 2 & 2 \\
\hline & Hymenolobium petraeum & 2 & & & 4 & 4 \\
\hline & Hymenolobium sericeum & 20 & 0,38 & 58,50 & 2 & 1 \\
\hline & Ormosia coccinea & 1 & & & 1 & 4 \\
\hline & Ormosia flava & 8 & 0,39 & 55,30 & 4 & 2 \\
\hline & Ormosia paraensis & 1 & 0,06 & 34,80 & 1 & 2 \\
\hline & Platymiscium spp & 7 & 0,35 & 52,50 & 4 & 2 \\
\hline & Platymiscium ulei & 3 & 0,34 & 40,70 & 4 & 2 \\
\hline & Pterocarpus rhorii & 1 & & & 1 & 4 \\
\hline & Pterocarpus spp & 2 & & & 2 & 4 \\
\hline & Vatairea erythrocarpa & 11 & 0,39 & 44,20 & 1 & 1 \\
\hline
\end{tabular}

FLORESTA, Curitiba, PR, v. 38, n. 1, jan./mar. 2008. 


\begin{tabular}{|c|c|c|c|c|c|c|}
\hline Família & Nome Científico & $\mathbf{N}$ & IPA & $\mathbf{P}_{95}$ & $\mathbf{G}$ & D \\
\hline & Vatairea spp & 30 & 0,39 & 57,30 & 2 & 1 \\
\hline & Vataireopsis speciosa & 3 & 0,12 & 120,30 & 1 & 2 \\
\hline \multirow{6}{*}{ FLACOURTIACEAE } & Casearia arborea & 1 & & & 1 & 4 \\
\hline & Casearia favitensis & 3 & 0,05 & 22 & 1 & 2 \\
\hline & Casearia pitumba & 3 & 0,32 & 21,50 & 4 & 2 \\
\hline & Homalium recemosum & 2 & & & 1 & 4 \\
\hline & Laetia procera & 32 & 0,29 & 53,10 & 2 & 1 \\
\hline & $\mathrm{Ni}$ & 2 & 0,53 & 22 & 4 & 2 \\
\hline \multirow[t]{4}{*}{ GUTTIFERAE } & Calophyllum brasiliensis & 2 & 0,14 & 64,20 & 1 & 2 \\
\hline & Platonia insignis & 4 & 0,04 & 107 & 1 & 2 \\
\hline & Symphonia globulifera & 41 & 0,46 & 56,30 & 2 & 1 \\
\hline & Tomovita cephalostigma & 8 & 0,18 & 25,50 & 1 & 2 \\
\hline HIPPOCRATEACEAE & Cheiloclinium cognatum & 2 & 0,23 & 20,60 & 3 & 2 \\
\hline \multirow{5}{*}{ HUMIRIACEAE } & Endopleura uchi & 41 & 0,55 & 82,10 & 3 & 1 \\
\hline & Saccoglottis amazonica & 2 & 0,56 & 28,40 & 4 & 2 \\
\hline & Saccoglottis guianensis & 1 & 0,43 & 30,50 & 4 & 2 \\
\hline & Saccoglottis $\mathrm{sp}$ & 1 & & & 1 & 4 \\
\hline & Vantanea guianensis & 3 & 0,35 & 21,50 & 4 & 2 \\
\hline \multirow[t]{3}{*}{ ICACINACEAE } & Dendrobangia boliviana & 199 & 0,24 & 45,70 & 1 & 0 \\
\hline & Emmotum fagifolium & 8 & 0,38 & 33,50 & 4 & 2 \\
\hline & Poraqueiba guianensis & 2 & 0,10 & 31,80 & 1 & 2 \\
\hline \multirow[t]{23}{*}{ LAURACEAE } & Aniba albescens & 45 & 0,35 & 35,10 & 1 & 1 \\
\hline & Aniba canellila & 5 & 0,18 & 66,50 & 2 & 2 \\
\hline & Aniba cayennensis & 2 & 0,18 & 21,70 & 1 & 2 \\
\hline & Aniba fragans & 11 & 0,25 & 55,90 & 2 & 1 \\
\hline & Aniba gigantofolia & 14 & 0,16 & 62,80 & 2 & 1 \\
\hline & Aniba paraensis & 1 & 0,32 & 26,40 & 4 & 2 \\
\hline & Aniba puchury & 1 & 0,10 & 22,30 & 1 & 2 \\
\hline & Aniba roseodora & 1 & 0,12 & 45,30 & 1 & 2 \\
\hline & Aniba squarenses & 1 & 0,13 & 36,50 & 1 & 2 \\
\hline & Beilschmiedia spp & 10 & 0,27 & 32,80 & 1 & 1 \\
\hline & Beilschwiedia spp & 30 & 0,54 & 30,90 & 4 & 1 \\
\hline & Licaria canella & 29 & 0,28 & 59,30 & 2 & 1 \\
\hline & Licaria reitzkleiniana & 1 & 0,33 & 27,20 & 4 & 2 \\
\hline & Mezilaurus lindaviana & 41 & 0,15 & 58 & 2 & 1 \\
\hline & Nectandra myriantha & 79 & 0,39 & 69,20 & 2 & 0 \\
\hline & $\mathrm{Ni}$ & 44 & 0,36 & 46,50 & 1 & 1 \\
\hline & Ocotea amazonica & 2 & 0,27 & 57,20 & 3 & 2 \\
\hline & Ocotea costulata & 8 & 0,33 & 27,70 & 4 & 2 \\
\hline & Ocotea dissimilis & 19 & 0,24 & 49,70 & 1 & 1 \\
\hline & Ocotea douradensis & 161 & 0,36 & 59 & 2 & 0 \\
\hline & Ocotea $\mathrm{spp}$ & 46 & 0,22 & 44,60 & 1 & 1 \\
\hline & Ocotea sprucei & 2 & 0,60 & 20,80 & 5 & 2 \\
\hline & Persea jariensis & 16 & 0,21 & 71,70 & 2 & 1 \\
\hline \multirow[t]{19}{*}{ LECYTHIDACEAE } & Bertholletia excels. & 2 & 0,51 & 145,80 & 4 & 2 \\
\hline & Corytophora rimosa & 115 & 0,27 & 72,30 & 2 & 0 \\
\hline & Couratari guianensis & 10 & 0,23 & 33,20 & 1 & 1 \\
\hline & Couratari oblongifolia & 1 & 0,15 & 41,70 & 4 & 2 \\
\hline & Couratarispp & 2 & 0,39 & 38 & 4 & 2 \\
\hline & Eschweilera mazônica & 103 & 0,22 & 41,90 & 1 & 0 \\
\hline & Eschweilera coriacea & 135 & 0,15 & 47 & 1 & 0 \\
\hline & Eschweilera floribunda & 1 & & & 1 & 4 \\
\hline & Eschweilera fracta & 8 & 0,11 & 25 & 1 & 2 \\
\hline & Eschweilera jurunensis & 219 & 0,24 & 54,60 & 2 & 0 \\
\hline & Eschweilera pecicellata & 1 & 0,42 & 42,50 & 4 & 2 \\
\hline & Eschweilera spp & 42 & 0,12 & 45 & 1 & 1 \\
\hline & Eschweilera subglandulosa & 10 & 0,10 & 27,60 & 1 & 1 \\
\hline & Gustavia augusta & 20 & 0,04 & 23,50 & 1 & 1 \\
\hline & Gustavia hexapetala & 50 & 0,31 & 64,20 & 2 & 0 \\
\hline & Lecythis lurida & 8 & 0,42 & 67,50 & 4 & 2 \\
\hline & Lecythis poitequi & 95 & 0,16 & 60,60 & 2 & 0 \\
\hline & Lecythis spp & 20 & 0,41 & 84,20 & 3 & 1 \\
\hline & Lecythis usitata & 4 & 0,19 & 40,30 & 1 & 2 \\
\hline & Antonia ovata & 2 & 0,16 & 21,70 & 1 & 2 \\
\hline \multirow{2}{*}{ MALPIGHIACEAE } & Byrsonima densa & 12 & 0,87 & 52,70 & 4 & 1 \\
\hline & Byrsonima stipulacea & 22 & 1,05 & 24,60 & 4 & 1 \\
\hline
\end{tabular}




\begin{tabular}{|c|c|c|c|c|c|c|}
\hline Família & Nome Científico & $\mathbf{N}$ & IPA & $\mathbf{P}_{95}$ & $\mathbf{G}$ & $\mathbf{D}$ \\
\hline \multirow[t]{9}{*}{ MELASTOMATACEAE } & Belucia dichotoma & 22 & 0,85 & 25,40 & 4 & 1 \\
\hline & Miconia amazonicum & 1 & & & 4 & 4 \\
\hline & Miconia guianensis & 1 & & & 4 & 4 \\
\hline & Miconia spp & 4 & & & 4 & 4 \\
\hline & Miconia surinamensis & 20 & 0,52 & 30,60 & 4 & 1 \\
\hline & Mouriri callocarpa & 92 & 0,23 & 53,20 & 2 & 0 \\
\hline & Mouriri sp & 1 & 0,12 & 20,20 & 1 & 2 \\
\hline & Mouriria brachyanthera & 4 & 0,16 & 55,30 & 1 & 2 \\
\hline & Mouriria spp & 20 & 0,20 & 47,10 & 1 & 1 \\
\hline \multirow[t]{6}{*}{ MELIACEAE } & Carapa guianensis & 28 & 0,33 & 64,50 & 2 & 1 \\
\hline & Guarea grandifolia & 1 & & & 1 & 4 \\
\hline & Guarea pebescens & 1 & & & 4 & 4 \\
\hline & $\mathrm{Ni}$ & 4 & 0,11 & 22,10 & 1 & 2 \\
\hline & Trichilia lecointei & 5 & 0,31 & 45,50 & 4 & 2 \\
\hline & Trichilia septentrionalis & 7 & 0,16 & 26 & 1 & 2 \\
\hline \multirow[t]{34}{*}{ MIMOSACEAE } & Abarema jupunba & 2 & & & 2 & 4 \\
\hline & Balizia elegans & 1 & 1,29 & 23,50 & 5 & 2 \\
\hline & Dinizia excelsa & 5 & 0,53 & 173,20 & 4 & 2 \\
\hline & Enterolobium barnebianum & 2 & 0,29 & 29,90 & 4 & 2 \\
\hline & Enterolobium schomburgkii & 9 & 0,52 & 101,20 & 4 & 2 \\
\hline & Inga alba & 48 & 0,74 & 69,50 & 5 & 1 \\
\hline & Inga bracteosa & 5 & 0,42 & 27,90 & 4 & 2 \\
\hline & Inga capitata & 59 & 0,61 & 34,40 & 4 & 0 \\
\hline & Inga cayennensis & 24 & 0,46 & 21 & 4 & 1 \\
\hline & Inga edulis & 3 & 0,70 & 23,30 & 4 & 2 \\
\hline & Inga heterophylla & 31 & 0,51 & 43,90 & 4 & 1 \\
\hline & Inga negrensis & 10 & & & 4 & 4 \\
\hline & Inga paraensis & 112 & 0,55 & 37 & 4 & 0 \\
\hline & Inga rubiginosa & 11 & 0,54 & 28,50 & 4 & 1 \\
\hline & Inga spp & 66 & 0,37 & 42 & 1 & 0 \\
\hline & Inga tarapotensis & 17 & & & 4 & 4 \\
\hline & Marmaroxylon racemosum & 22 & 0,25 & 53 & 2 & 1 \\
\hline & $\mathrm{Ni}$ & 1 & & & 4 & 4 \\
\hline & Parkia decussata & 19 & 1,11 & 83,50 & 5 & 1 \\
\hline & Parkia gigantocarpa & 1 & 0,26 & 20,80 & 3 & 2 \\
\hline & Parkia oppositifolia & 11 & 0,70 & 64,20 & 5 & 1 \\
\hline & Parkia pendula & 11 & 0,65 & 104,50 & 3 & 1 \\
\hline & Parkia reticulata & 2 & 0,40 & 21,20 & 4 & 2 \\
\hline & Parkia ulei & 32 & 1,16 & 86,80 & 5 & 1 \\
\hline & Pithecellobium cochleatum & 35 & 0,22 & 37,90 & 1 & 1 \\
\hline & Pithecellobium decandrum & 31 & 0,51 & 68,30 & 2 & 1 \\
\hline & Pithecellobium pedicellare & 1 & 0,47 & 71,40 & 4 & 2 \\
\hline & Pithecellobium spp & 8 & 0,54 & 44,40 & 4 & 2 \\
\hline & Pseudopiptadenia psilostachya & 50 & 0,65 & 102,80 & 3 & 0 \\
\hline & Stryphnodendron paniculatum & 42 & 0,55 & 56,60 & 2 & 1 \\
\hline & Stryphnodendron pulcherrimum & 27 & 0,97 & 59,60 & 5 & 1 \\
\hline & Stryphnodendron spruceana & 1 & & & 2 & 4 \\
\hline & Zygia ampla & 1 & 0,01 & 24 & 1 & 2 \\
\hline & Zygia latifolia & 4 & & & 4 & 1 \\
\hline \multirow[t]{3}{*}{ MONIMIACEAE } & Siparuna decipiens & 11 & 0,08 & 31,70 & 1 & 1 \\
\hline & Siparuna guianensis & 33 & 0,08 & 31,50 & 1 & 1 \\
\hline & Siparuna sp & 1 & 0,06 & 25,50 & 1 & 2 \\
\hline \multirow[t]{14}{*}{ MORACEAE } & Brosimum acutifolium & 23 & 0,25 & 81,80 & 3 & 1 \\
\hline & Brosimum guianensis & 51 & 0,14 & 35 & 1 & 0 \\
\hline & Brosimum lactescens & 12 & 0,11 & 44,70 & 1 & 1 \\
\hline & Brosimum parinarioides & 27 & 0,41 & 70,40 & 2 & 1 \\
\hline & Brosimum rubescens & 28 & 0,30 & 52,40 & 2 & 1 \\
\hline & Brosimum spp & 29 & 0,14 & 43,80 & 1 & 1 \\
\hline & Cecropia obtusa & 33 & 0,30 & 27 & 1 & 1 \\
\hline & Cecropia sciadophylla & 235 & 0,90 & 38,70 & 4 & 0 \\
\hline & Cecropia spp & 2 & 0,16 & 26,80 & 1 & 2 \\
\hline & Clarisia racemosa & 8 & 0,32 & 78,30 & 4 & 2 \\
\hline & Helicostylis spp & 23 & 0,12 & 32,20 & 1 & 1 \\
\hline & Maquira guianensis & 54 & 0,24 & 37,50 & 1 & 0 \\
\hline & Maquira sclerophylla & 3 & 0,15 & 25,40 & 1 & 2 \\
\hline & Maquira spp & 7 & 0,19 & 39,80 & 2 & 2 \\
\hline
\end{tabular}

FLORESTA, Curitiba, PR, v. 38, n. 1, jan./mar. 2008. 


\begin{tabular}{|c|c|c|c|c|c|c|}
\hline Família & Nome Científico & $\mathbf{N}$ & IPA & $\mathbf{P}_{95}$ & $\mathbf{G}$ & D \\
\hline & $\mathrm{Ni}$ & 3 & 0,17 & 22,80 & 3 & 2 \\
\hline & Perebea mollis & 1 & 0,08 & 20,40 & 1 & 2 \\
\hline & Pourouma miror & 13 & 0,64 & 24,30 & 4 & 1 \\
\hline & Pourouma spp & 22 & 0,61 & 22,95 & 4 & 1 \\
\hline & Pourouma vilosa & 47 & 0,54 & 33 & 4 & 1 \\
\hline & Trymotococus paraensis & 10 & 0,12 & 34,90 & 1 & 1 \\
\hline \multirow[t]{8}{*}{ MYRISTICACEAE } & Iryanthera sagotiana & 148 & 0,22 & 43,50 & 1 & 0 \\
\hline & Iryanthera spp & 4 & 0,07 & 31,80 & 1 & 2 \\
\hline & Myrciaria floribunda & 45 & 0,15 & 35,80 & 1 & 1 \\
\hline & Osteophloeum platyspermum & 5 & 0,75 & 51,60 & 5 & 2 \\
\hline & Virola calophylla & 2 & 0,16 & 20,70 & 1 & 2 \\
\hline & Virola michelli & 167 & 0,46 & 61,10 & 2 & 0 \\
\hline & Virola sebifera & 2 & 0,41 & 27,10 & 4 & 2 \\
\hline & Virola spp & 6 & 0,14 & 27,60 & 1 & 2 \\
\hline \multirow[t]{7}{*}{ MYRTACEAE } & Eugenia paraensis & 1 & 0,24 & 21,70 & 3 & 2 \\
\hline & Eugenia patrisii & 12 & 0,11 & 28,50 & 1 & 1 \\
\hline & Myrcia clusiifolia & 2 & 0,11 & 20,70 & 1 & 2 \\
\hline & Myrcia falax & 71 & 0,10 & 33,30 & 1 & 0 \\
\hline & Myrciaria floribunda & 3 & 0,13 & 24,20 & 1 & 2 \\
\hline & $\mathrm{Ni}$ & 4 & 0,13 & 29,20 & 1 & 2 \\
\hline & $\mathrm{Ni}$ & 138 & 0,20 & 70,90 & 2 & 0 \\
\hline \multirow[t]{2}{*}{ NYCTAGINACEAE } & Neea constricta & 81 & 0,22 & 41,90 & 1 & 0 \\
\hline & Neea $\mathrm{spp}$ & 3 & 0,32 & 25,80 & 4 & 2 \\
\hline OCHANACEAE & Ouratea polygyna & 8 & 0,07 & 27 & 1 & 2 \\
\hline \multirow[t]{5}{*}{ OLACACEAE } & Chaunochiton kappleri & 15 & 0,30 & 70,70 & 2 & 1 \\
\hline & Douradoa consimilis & 1 & 0,16 & 26,30 & 1 & 2 \\
\hline & Dulacia guianensis & 3 & 0,11 & 36,90 & 1 & 2 \\
\hline & Minquartia guianensis & 167 & 0,25 & 71 & 2 & 0 \\
\hline & $\mathrm{Ni}$ & 2 & 0,08 & 39,80 & 1 & 2 \\
\hline OPILIACEAE & Agonandra brasiliensis & 11 & 0,10 & 43,30 & 1 & 1 \\
\hline \multirow[t]{4}{*}{ PAPILIONOIDEAE } & Acosmium nitens & 1 & 0,05 & 21 & 1 & 2 \\
\hline & Platymiscium trinitatis & 1 & 0,33 & 26,80 & 4 & 2 \\
\hline & Platysmicium spp & 4 & 0,29 & 39,20 & 3 & 2 \\
\hline & Pterocarpus rohrii & 1 & & & 1 & 4 \\
\hline QUIINACEAE & Lacunaria spruceana & 5 & 0,16 & 22,40 & 1 & 2 \\
\hline \multirow[t]{8}{*}{ RUBIACEAE } & Borojoa sorbilis & 2 & 0,17 & 25,40 & 2 & 2 \\
\hline & Chimarrhis turbinata & 51 & 0,31 & 54 & 2 & 0 \\
\hline & Duroia macrophylla & 1 & & & 2 & 4 \\
\hline & Duroia $\mathrm{sp}$ & 1 & 0 & 25,60 & 1 & 2 \\
\hline & Ferdinandusa elliptica & 2 & 0,25 & 22,20 & 3 & 2 \\
\hline & Ferdinandusa paraensis & 1 & & & 3 & 4 \\
\hline & $\mathrm{Ni}$ & 6 & 0,18 & 42,40 & 1 & 2 \\
\hline & Psychotria mapourioides & 6 & 0,07 & 22,70 & 1 & 2 \\
\hline \multirow[t]{2}{*}{ RUTACEAE } & Zanthoxylum panamensis & 1 & 0,74 & 48,70 & 5 & 2 \\
\hline & Zanthoxylum spp & 2 & 0,20 & 38,10 & 3 & 2 \\
\hline \multirow[t]{4}{*}{ SAPINDACEAE } & Cupania cf. Hirsuta & 15 & 0,19 & 33,80 & 1 & 1 \\
\hline & Toulicia acutifolia & 8 & 0,17 & 37,10 & 1 & 2 \\
\hline & Toulicia bulata & 1 & 0,59 & 26,20 & 4 & 2 \\
\hline & Vouarana guianensis & 1 & 0,47 & 25,30 & 4 & 2 \\
\hline \multirow[t]{17}{*}{ SAPOTACEAE } & Achrouteria pamifera & 3 & 0,22 & 48,20 & 3 & 2 \\
\hline & Barylucuma decusata & 3 & 0,21 & 27,30 & 2 & 2 \\
\hline & Eclinusa guianensis & 15 & 0,31 & 54,20 & 2 & 1 \\
\hline & Manilkara bidentada & 108 & 0,28 & 88,20 & 3 & 0 \\
\hline & Manilkara huberi & 247 & 0,42 & 93,30 & 3 & 0 \\
\hline & Micropholis guianensis & 147 & 0,31 & 65,70 & 2 & 0 \\
\hline & Micropholis mensalis & 2 & 0,21 & 59,70 & 4 & 2 \\
\hline & Micropholis venulosa & 2 & 0,11 & 36,50 & 1 & 2 \\
\hline & Nemaluma anomalum & 11 & 0,23 & 50,10 & 2 & 1 \\
\hline & Nemaluma engleri & 2 & 0,27 & 63,10 & 3 & 2 \\
\hline & Nemaluma spp & 2 & & & 2 & 4 \\
\hline & $\mathrm{Ni}$ & 446 & 0,21 & 54,90 & 2 & 0 \\
\hline & Pouteria amapaensis & 2 & 0,35 & 40,30 & 4 & 2 \\
\hline & Pouteria amazônica & 13 & 0,22 & 60,50 & 2 & 1 \\
\hline & Pouteria anomala & 1 & & & 2 & 4 \\
\hline & Pouteria bilocularis & 5 & 0,39 & 45,30 & 4 & 2 \\
\hline & Pouteria branquetum & 2 & 0,26 & 22,30 & 3 & 2 \\
\hline
\end{tabular}




\begin{tabular}{|c|c|c|c|c|c|c|}
\hline Família & Nome Científico & $\mathbf{N}$ & IPA & $\mathbf{P}_{95}$ & $\mathbf{G}$ & D \\
\hline & Pouteria caldense & 1 & & & 1 & 4 \\
\hline & Pouteria cladantha & 16 & 0,23 & 51,20 & 2 & 1 \\
\hline & Pouteria crassifolia & 5 & 0,36 & 52,10 & 4 & 2 \\
\hline & Pouteria elegans & 14 & 0,17 & 75 & 2 & 1 \\
\hline & Pouteria guianensis & 2 & 0,18 & 49,90 & 1 & 2 \\
\hline & Pouteria kruko & 32 & 0,32 & 57,50 & 2 & 1 \\
\hline & Pouteria lasiocarpa & 2 & 0,28 & 30,30 & 4 & 2 \\
\hline & Pouteria laurifolia & 53 & 0,20 & 43,90 & 1 & 0 \\
\hline & Pouteria macrocarpa & 53 & 0,16 & 61,70 & 2 & 0 \\
\hline & Pouteria spp & 408 & 0,24 & 61,10 & 2 & 0 \\
\hline & Pouteria oblanceolata & 10 & 0,08 & 47,50 & 1 & 1 \\
\hline & Pouteria oppositifolia & 1 & & & 2 & 4 \\
\hline & Pouteria pariri & 6 & 0,51 & 64,80 & 4 & 2 \\
\hline & Pouteria procera & 3 & 0,30 & 22 & 4 & 2 \\
\hline & Pouteria spp & 54 & 0,21 & 61 & 2 & 0 \\
\hline & Pouteria spruceana & 7 & 0,21 & 31,90 & 3 & 2 \\
\hline & Prieurella amapaensis & 7 & 0,29 & 42,80 & 3 & 2 \\
\hline & Prieurella amazonicum & 1 & 0,31 & 23,90 & 4 & 2 \\
\hline & Prieurella sp & 1 & 0,12 & 48 & 1 & 2 \\
\hline & Prieurella preurii & 38 & 0,25 & 57,80 & 2 & 1 \\
\hline & Ragala balata & 1 & 0,17 & 23,80 & 1 & 2 \\
\hline & Richardella rodriguesiana & 10 & 0,28 & 55,10 & 2 & 1 \\
\hline & Syzygiopsis amazonica & 5 & 0,18 & 52 & 1 & 2 \\
\hline & Syzygiopsis oppositifolia & 115 & 0,31 & 87,90 & 3 & 0 \\
\hline & Syzygiopsis pachycarpa & 53 & 0,29 & 65,30 & 2 & 0 \\
\hline & Syzygiopsis spruceana & 1 & 0,50 & 53,90 & 4 & 2 \\
\hline \multirow[t]{2}{*}{ SIMAROUBACEAE } & Simaba multiflora & 1 & 0,48 & 21 & 4 & 2 \\
\hline & Simaruba amara & 20 & 0,55 & 51 & 4 & 1 \\
\hline \multirow[t]{4}{*}{ STERCULIACEAE } & Sterculia excelsa var. pilosa & 72 & 0,24 & 41,60 & 1 & 0 \\
\hline & Sterculia $\mathrm{spp}$ & 2 & & & 1 & 4 \\
\hline & Theobroma subincanum & 4 & 0 & 21,50 & 1 & 2 \\
\hline & Theobroma sylvestre & 1 & 0,16 & 23,20 & 2 & 2 \\
\hline STYRACACEAE & Styrax sieberi & 4 & 2 & 25,70 & 5 & 2 \\
\hline \multirow[t]{2}{*}{ TILIACEAE } & Apeiba burchellii & 39 & 0,41 & 62,30 & 2 & 1 \\
\hline & Luehea speciosa & 13 & 0,29 & 73,10 & 2 & 1 \\
\hline ULMACEAE & Ampelocera edentula & 22 & 0,28 & 34,20 & 1 & 1 \\
\hline VERBENACEAE & Vitex triflora & 1 & 0,11 & 24 & 1 & 2 \\
\hline \multirow[t]{2}{*}{ VIOLACEAE } & Rinorea guianensis & 61 & 0,18 & 39,40 & 1 & 0 \\
\hline & Rinorea lindeniana & 54 & 0,08 & 25,20 & 1 & 0 \\
\hline \multirow[t]{12}{*}{ VOCHYSIACEAE } & Erisma laurifolium & 1 & 0,56 & 41,70 & 5 & 2 \\
\hline & Erisma uncinatum & 3 & 0,40 & 105,50 & 4 & 2 \\
\hline & $\mathrm{Ni}$ & 2 & 0,36 & 48,40 & 4 & 2 \\
\hline & Qualea albiflora & 55 & 0,63 & 78 & 5 & 0 \\
\hline & Qualea coerulea & 1 & 0,14 & 20,40 & 1 & 2 \\
\hline & Qualea paraensis & 17 & 0,49 & 53,80 & 2 & 1 \\
\hline & Qualea rosea & 2 & 0,23 & 20,90 & 3 & 2 \\
\hline & Vochysia eximia & 1 & & & 4 & 4 \\
\hline & Vochysia guianensis & 10 & 0,57 & 72,60 & 2 & 1 \\
\hline & Vochysia $\mathrm{sp}$ & 1 & 0,77 & 43,20 & 5 & 2 \\
\hline & Vochysia obscura & 7 & 0,69 & 51 & 4 & 2 \\
\hline & Vochysia vismiaefolia & 1 & 0,64 & 41 & 4 & 2 \\
\hline
\end{tabular}

\section{Influência dos tratamentos no agrupamento de espécies}

Os números totais de espécies incluídas nesta análise foram similares para cada tratamento, à exceção da testemunha (T0), mas as proporções de espécies em cada grupo de incremento de diâmetro variaram entre o tratamento T0 e os demais tratamentos: $38,26 \%$ das espécies do tratamento-controle foram designadas, por exemplo, para grupo de incremento de diâmetro muito lento, mas para as espécies sob tratamentos de exploração/desbastes esses valores variaram de 28,65 a 36,94\%. Semelhantemente, enquanto que a proporção de espécies no tratamento T0 foi maior no Grupo 1, para os demais tratamentos, sob exploração e refinamento/liberação, a proporção maior foi para o Grupo 2 (Tabela 6).

A soma das proporções das espécies nos grupos de incremento moderado, rápido e muito rápido foi de $24,18 \%$ para o controle e de $27,27 \%, 30,68 \%$ e $30,22 \%$ para as espécies sob tratamento leve, médio e pesado, respectivamente. O número de espécies de crescimento muito lento, critério para inclusão nos agrupamentos, foi reduzido pelos tratamentos silviculturais. 
Embora as proporções de espécies em diferentes grupos de incremento de diâmetro tenham diferido entre tratamentos, estes foram semelhantes no tamanho e proporção do grupo de incrementos moderado e muito rápido.

Tabela 6. Número e proporções de espécies em cada agrupamento ecológico de espécies por tratamento silvicultural.

Table 6. Number and proportions of species in each species ecological grouping for different silvicultural treatment.

\begin{tabular}{|c|c|c|c|c|c|c|c|c|c|c|c|c|c|}
\hline \multirow{2}{*}{ Grupo } & \multicolumn{13}{|c|}{ Número e proporção de espécies por tratamento } \\
\hline & T0 & T1 & T2 & T3 & T4 & T5 & T6 & T7 & T8 & T9 & T10 & T11 & T12 \\
\hline \multirow[b]{2}{*}{1} & 386 & 270 & 228 & 208 & 222 & 223 & 211 & 212 & 241 & 235 & 262 & 228 & 224 \\
\hline & $(38,26)$ & $(36,94)$ & $(31,45)$ & $(28,49)$ & $(29,88)$ & $(30,30)$ & $(28,71)$ & $(28,65)$ & $(32,74)$ & $(30,52)$ & $(35,12)$ & $(29,27)$ & $(28,90)$ \\
\hline 2 & $(37,56)$ & $(38,03)$ & $(41,52)$ & $(43,42)$ & $(41,18)$ & $(37,50)$ & $(38,10)$ & $(41,89)$ & $(39,40)$ & $(40,91)$ & $(31,37)$ & $(41,08)$ & $(41,94)$ \\
\hline \multirow{2}{*}{3} & 123 & 90 & 90 & 100 & 96 & 104 & 92 & 107 & 93 & 91 & 98 & 97 & 89 \\
\hline & $(12,19)$ & $(12,31)$ & $(12,41)$ & $(13,70)$ & $(12,92)$ & $(14,13)$ & $(12,52)$ & $(14,46)$ & $(12,64)$ & $(11,82)$ & $(13,14)$ & $(12,45)$ & $(11,48)$ \\
\hline 4 & 67 & 60 & 65 & 62 & 77 & 101 & 94 & 72 & 70 & 73 & 112 & 93 & 92 \\
\hline 5 & $(5,35)$ & $(4,51)$ & $(5,66)$ & $(5,89)$ & $(5,65)$ & $(4,35)$ & $(7,89)$ & $(5,27)$ & $(5,71)$ & $(7,27)$ & $(5,36)$ & $(5,26)$ & $(5,81)$ \\
\hline Total & $\begin{array}{r}1009 \\
(100)\end{array}$ & $\begin{array}{r}731 \\
(100)\end{array}$ & $\begin{array}{r}725 \\
(100)\end{array}$ & $\begin{array}{r}730 \\
(100)\end{array}$ & $\begin{array}{r}743 \\
(100)\end{array}$ & $\begin{array}{r}736 \\
(100)\end{array}$ & $\begin{array}{r}735 \\
(100)\end{array}$ & $\begin{array}{r}740 \\
(100)\end{array}$ & $\begin{array}{r}736 \\
(100)\end{array}$ & $\begin{array}{r}770 \\
(100)\end{array}$ & $\begin{array}{r}746 \\
(100)\end{array}$ & $\begin{array}{r}779 \\
(100)\end{array}$ & $\begin{array}{r}775 \\
(100)\end{array}$ \\
\hline
\end{tabular}

Os tratamentos foram significativamente diferentes em relação aos incrementos dentro dos grupos pelo teste de Tukey no nível de 1\% de probabilidade (Tabela 7). Observa-se que, nos grupos de incrementos muito lento e lento, o povoamento sem interferência mostrou as menores médias, estatisticamente diferentes das dos demais tratamentos. Nos grupos de incrementos moderado, rápido e muito rápido, as médias foram muito similares e não diferiram estatisticamente das do povoamento sem interferência.

Seguiu-se Vanclay (1994), Alder (1995) e Phillips et. al. (2001) na busca de uma classificação não-subjetiva de espécies, com base no incremento em diâmetro observado e alguma medida de tamanho, neste caso, o percentil $95 \%$ da distribuição cumulativa dos diâmetros.

Tabela 7. Variação da taxa de crescimento por tratamento para cada agrupamento. Valores seguidos da mesma letra para o mesmo grupo não diferem entre si pelo teste de Tukey no nível $1 \%$ de probabilidade.

Table 7. Variation in growth rate per treatment for each species group. Values followed by the same letter within the same group do not differ by the test of Tukey to probability level of $1 \%$.

\begin{tabular}{llllllllllll}
\hline Tratamento & Grupo 1 & \multicolumn{1}{l}{ Grupo 2 } & \multicolumn{2}{l}{ Grupo 3 } & \multicolumn{2}{l}{ Grupo 4 } & \multicolumn{2}{c}{ Grupo 5 } \\
\hline 0 & 0,13 & $\mathrm{c}$ & 0,18 & $\mathrm{e}$ & 0,32 & $\mathrm{ab}$ & 0,41 & $\mathrm{~b}$ & 0,86 & $\mathrm{ab}$ \\
1 & 0,18 & $\mathrm{~b}$ & 0,24 & $\mathrm{~cd}$ & 0,32 & $\mathrm{ab}$ & 0,56 & $\mathrm{ab}$ & 0,93 & $\mathrm{ab}$ \\
4 & 0,19 & $\mathrm{~b}$ & 0,23 & $\mathrm{~d}$ & 0,33 & $\mathrm{ab}$ & 0,43 & $\mathrm{~b}$ & 1,01 & $\mathrm{ab}$ \\
6 & 0,19 & $\mathrm{~b}$ & 0,29 & $\mathrm{ab}$ & 0,37 & $\mathrm{ab}$ & 0,66 & $\mathrm{a}$ & 0,98 & $\mathrm{ab}$ \\
5 & 0,21 & $\mathrm{ab}$ & 0,26 & $\mathrm{bcd}$ & 0,31 & $\mathrm{~b}$ & 0,56 & $\mathrm{ab}$ & 1,12 & $\mathrm{a}$ \\
8 & 0,21 & $\mathrm{ab}$ & 0,28 & $\mathrm{abc}$ & 0,34 & $\mathrm{ab}$ & 0,64 & $\mathrm{a}$ & 1,04 & $\mathrm{ab}$ \\
2 & 0,22 & $\mathrm{ab}$ & 0,29 & $\mathrm{ab}$ & 0,34 & $\mathrm{ab}$ & 0,45 & $\mathrm{ab}$ & 0,78 & $\mathrm{~b}$ \\
3 & 0,22 & $\mathrm{ab}$ & 0,26 & $\mathrm{bcd}$ & 0,36 & $\mathrm{ab}$ & 0,54 & $\mathrm{ab}$ & 0,87 & $\mathrm{ab}$ \\
7 & 0,22 & $\mathrm{ab}$ & 0,28 & $\mathrm{abc}$ & 0,31 & $\mathrm{ab}$ & 0,58 & $\mathrm{ab}$ & 1,01 & $\mathrm{ab}$ \\
11 & 0,22 & $\mathrm{ab}$ & 0,29 & $\mathrm{ab}$ & 0,39 & $\mathrm{ab}$ & 0,57 & $\mathrm{ab}$ & 1,05 & $\mathrm{ab}$ \\
9 & 0,23 & $\mathrm{ab}$ & 0,31 & $\mathrm{a}$ & 0,32 & $\mathrm{ab}$ & 0,58 & $\mathrm{ab}$ & 0,85 & $\mathrm{ab}$ \\
10 & 0,25 & $\mathrm{a}$ & 0,30 & $\mathrm{ab}$ & 0,41 & $\mathrm{a}$ & 0,53 & $\mathrm{ab}$ & 1,10 & $\mathrm{ab}$ \\
12 & 0,25 & $\mathrm{a}$ & 0,30 & $\mathrm{ab}$ & 0,35 & $\mathrm{ab}$ & 0,57 & $\mathrm{ab}$ & 1,01 & $\mathrm{ab}$ \\
\hline
\end{tabular}

$\mathrm{Na}$ figura 3 é mostrada a variação do ingresso por intervalo de monitoramento para cada agrupamento ecológico de espécies. Os maiores valores de ingresso e mortalidade são observados nos grupos 1 (crescimento muito lento, dossel inferior) e 2 (crescimento lento, dossel médio). Tanto o ingresso quanto a mortalidade podem ser explicados pela atividade de exploração e de tratamentos silviculturais (SILVA, 1989). 
$\mathrm{Na}$ exploração florestal, a abertura do dossel, causada pela queda das árvores vivas, parece ter favorecido o crescimento das árvores desses dois grupos que, geralmente, ocupam os estratos inferior e médio da floresta durante muito tempo, à espera de condições de luminosidade ideais para o seu crescimento.

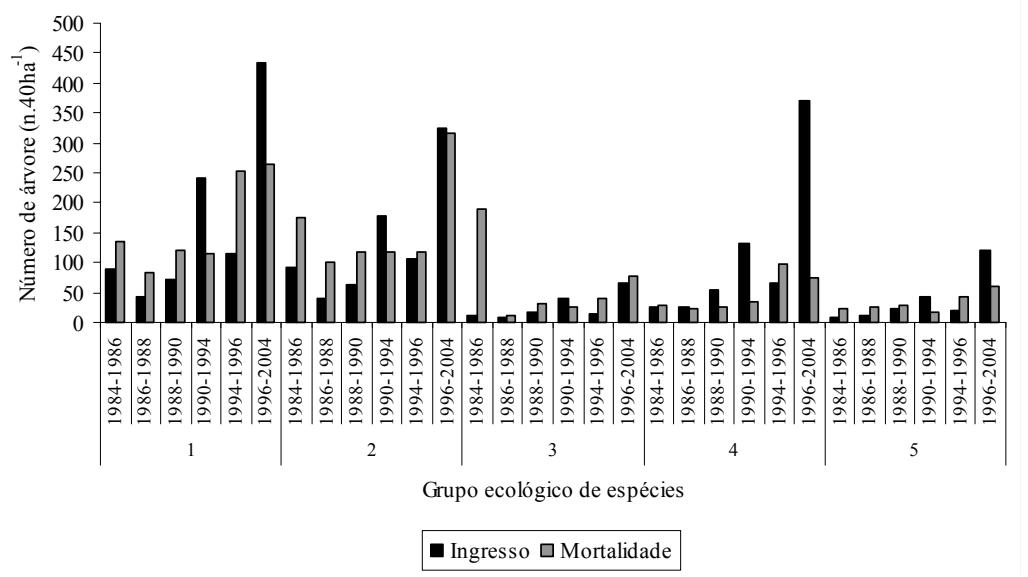

Figura 3. Ingresso e mortalidade nos cinco agrupamentos formados por intervalo de monitoramento para os 40 hectares amostrados na Floresta da Jari.

Figure 3. Ingrowth and mortality in the five groupings formed by interval of monitoring for the 40 hectares measured in the Jari forest.

$\mathrm{Na}$ exploração florestal, a queda da árvore é brusca e causa danos e morte no povoamento remanescente, principalmente nos extratos inferior e médio (Grupos 1 e 2). O crescimento de espécies pioneiras (Grupo 4) não foi favorecido pela exploração. O grande número de árvores mortas que se observou no período 1984-1986 para o Grupo 3 se deve à exploração florestal de espécies comerciais (contadas como mortas devido à exploração) que predominam nesse grupo.

As posições relativas das espécies nos agrupamentos e a autocorrelação das taxas de crescimento medidas em períodos de tempo sucessivos (SWAINE et al., 1987) sugerem que, embora os incrementos absolutos possam variar com o passar do tempo, mudanças na posição das espécies são improváveis.

O método de análise de agrupamento (cluster analisys) foi bastante eficiente na formação dos grupos. Formação dos grupos com base na autofreqüência das espécies assegurou que a distinção entre grupos de espécies refletiu diferenças reais. Agruparam-se 427 espécies em 5 grupos.

\section{CONCLUSÕES}

Com base nos resultados obtidos, conclui-se que:

- A exploração das árvores comerciais e a aplicação dos tratamentos silviculturais estimularam o ingresso de árvores dos grupos 1 (crescimento muito lento, dossel inferior) e 2 (crescimento lento, dossel médio) e, diferente do esperado, não favoreceram o crescimento de espécies pioneiras (grupo 4).

- O número de árvores de crescimento muito lento, critério para inclusão nos agrupamentos, foi reduzido pela aplicação dos tratamentos silviculturais.

- O método de análise de agrupamento (cluster analysis) foi bastante eficiente na formação dos grupos.

\section{AGRADECIMENTOS}

Ao Grupo Orsa, pelo apoio logístico na coleta dos dados.

\section{REFERÊNCIAS}

ALDER, D. Growth Modelling for Mixed Tropical Forests. Oxford: Department of Plant Sciences, University of Oxford, 1995. 231 p. (Tropical Forestry Paper, n. 30). 
ALDER, D.; OAVIKA, F.; SANCHEZ, M.; SILVA, J. N. M.; van der HOUT, P.; WRIGHT, H. L. Comparison of species growth rates using increment-size ordination. International Forestry Review, United Kingdom, v. 4, n. 3, p. 196-205, 2002.

ALDER, D; SILVA, J. N. M. An empirical cohort model for the management of Terra Firme forests in the Brazilian Amazon. Forest Ecology and Management, Amsterdan, v. 130, n.1-3 , p. 141-157, 2000.

ATTA-BOATENG, J.; MOSER, J. W. J. R. A method for classifying commercial tree species of an uneven-aged mixed species tropical forest for growth and yield model construction. Forest Ecology and Management, Amsterdan, v. 104, n. 1-3, p. 89-99, 1998.

BRAGA, P. I. S. Subdivisão fitogeográfica, tipos de vegetação, conservação e inventário florístico da floresta Amazônica. Acta Amazônica. Suplwemento, Manaus, v. 15, p. 53-80, 1979.

BUDOWSKI, G. Distribution of tropical american rain forest species in the light of successional processes. Turrialba, San Jose, CR, v. 15, p. 440-442, 1965.

DENSLOW, J. S. Gap partitioning among tropical rain forest trees. Biotropica, Washington, DC, v. 12, p. 47-55, 1980.

EBA'A ATYI, R. 1997. Potentials of cluster analysis for the aggregation of tree species from African tropical forests. In: IUFRO CONFERENCE ON GROWTH STUDIES IN TROPICAL MOIST FORESTS IN AFRICA, 1996. Proceedings... Kumasi: Forestry Research Institute of Ghana, 1997. p $170-176$.

FINEGAN, B The management potential of neotropical secundary lowland rain forest. Forest Ecology and Management, Amsterdan, v. 47, v. 2, p. 295 - 321, 1992.

FINEGAN, B.; CAMACHO, M.; ZAMORA, N. Diameter increment patterns among 106 tree species in a logged and silviculturally treated Costa Rican rain forest. Forest Ecology and Management, Amsterdan, v. 121, n. 3, p. 159-176, 1999.

HARTSHORN, G. S. Neotropical forest dynamics. Biotropica, Washington, DC, v. 12, p. 23-30, 1980.

HIGUCHI, N.; JARDIM, F. C. S.; SANTOS, J.; ALENCAR, J. C. Bacia 3 - Inventário diagnóstico da regeneração natural. Acta Amazônica, Manuas, v. 15, p. 199-233, 1985.

LLERAS, E.; LEITE, A. M. C. A Biodiversidade amazônica sem mitos. Belém: Embrapa Amazônia Ocidental 2005. 20 p. (Série Documentos, n. 36).

PHILLIPS, P. D. et al. Grouping tree species for analysis of forest data in Kalimantan (Indonesian Borneo). Forest Ecology and Management, , Amsterdan, v. 157, n., p. 205-216, 2001.

PHILLIPS, P. D. et al. An ecological model for the management of natural forest in the Tapajos region, Amazonian Brazil. Edinburgh: The University of Edinburgh, 2002. 33 p. (SYMFOR Technical Note Series, n. 12). Disponível em: <http://www.symfor.org/technical/indez.html $>$. Acesso em: 01/08/2004.

PHILLIPS, P. D. et al. An individual-based spatially explicit simulation model for strategic forest management planning in the eastern Amazon. Ecological Modelling, Amsterdan, v. 173, p. 335-354, 2004.

RANKIN-DE-MERONA, et al. Preliminary results of a large-scale tree inventory of upland rain forest in the Central Amazon. Acta Amazônica, Manaus, v. 22, p. 493-534, 1992.

RIBEIRO, J. E. L. S. et al. Flora da Reserva Ducke: guia de identificação das plantas vasculares de uma floresta de terra firme na Amazônia Central. Manaus: INPA/DFID, 1999. 816 p.

SILVA, J. N. M. The behaviour of the tropical rain forest of the Brazilian Amazon after logging. 303 p. PhD Thesis - University of Oxford, UK, Oxford, 1989. 
SILVA, J. N. M.; LOPES, J. C. A. Inventário florestal contínuo em florestas tropicais: a metodologia utilizada pela EMBRAPA-CPATU na Amazônia brasileira. Belém: Embrapa-CPATU, 1984, 36 p. (Embrapa-CPATU. Documentos, n. 33).

SWAINE. M. D.; HALL, J. B.; ALEXANDER, I. J. Tree populations dynamics at Kade, Ghana (19681982). Journal of Tropical Ecology, Cambridge, v. 3, p. 331-345, 1987.

SWAINE. M. D.; WHITMORE, T. C. On the definition of ecological groups in tropical rain forest. Vegetatio. Acta geobotânica, The Hague, v. 75, p. 81-86, 1988.

VANCLAY, J. K. Aggregating tree species to develop diameter increment equations for tropical rainforests. Forest Ecology and Management, Amsterdan, v. 42, n.3-4, p. 143-168, 1991.

VANCLAY, J. K. Modeling forest growth and yield: applications to mixed tropical forests. Oxfordshire: CAB International, 1994. 312p. 